\title{
Identification of dominant error parameters in spectra measured by a TDEMI system
}

\author{
Miroslav Kamenský, Karol Kováč \\ Slovak University of Technology in Bratislava, Faculty of Electrical Engineering and Information Technology, Institute of Electrical \\ Engineering, Ilkovičova 3, 81219 Bratislava, Slovak Republic
}

\begin{abstract}
Multiresolution analog-to-digital converters (MRADC) are usually used in ime Domain ElectroMagnetic Interference (TDEMI) measuring systems for very fast signal sampling with a sufficient dynamic range. The properties of the spectrum measured by the TDEMI system influenced by imperfections in the MRADC are analyzed in this paper. Errors are caused by imperfect matching of the offset and gain and phase of the circuits used in parallel input channels typical for the MRADC. For deep analyses of MRADC behavior, a precise mathematical model has been created using the concept of additive error pulses. Furthermore, a dedicated process of the identification of discrepancy parameters from experimental data is proposed. Identified parameters enter the expressions of the model and enable side to side comparison of experimental and theoretical results.
\end{abstract}

\section{Section: RESEARCH PAPER}

Keywords: Multiresolution quantization, time domain EMI measurement, offset and slope errors, spectrum measurement, error identification

Citation: Miroslav Kamenský, Karol Kováč, Identification of dominant error parameters in spectra measured by a TDEMI system, Acta IMEKO, vol. 4, no. 1, article 10, February 2015, identifier: IMEKO-ACTA-04 (2015)-01-10

Editor: Paolo Carbone, University of Perugia

Received December $16^{\text {th }}, 2013$; In final form November $15^{\text {th }}, 2014$; Published February 2015

Copyright: (C) 2014 IMEKO. This is an open-access article distributed under the terms of the Creative Commons Attribution 3.0 License, which permits unrestricted use, distribution, and reproduction in any medium, provided the original author and source are credited

Funding: This work was supported by the Slovak Research and Development Agency under grant No. APVV-0333-11 and by the Slovak Grant Agency VEGA under grant No. 1/0963/12

Corresponding author: Miroslav Kamenský, e-mail: miroslav.kamensky@stuba.sk

\section{INTRODUCTION}

Special measuring systems are being developed for different areas of industry improving quality in many aspects. Today we often require an automated and fast measuring procedure alongside with high accuracy. Measuring of the electromagnetic interference (EMI) spectra has been obviously a time consuming procedure. Conventional analog EMI receivers are based on a superheterodyne principle. Here, only one narrow frequency band is transferred to the detector at a time via an intermediate frequency amplifier. Therefore timeconsuming sweeping through the whole measured frequency range is needed and several tens of minutes are often required to complete the whole EMI spectrum measurement. The advantage of such an arrangement is the high dynamic range of the spectrum measurement required by EMI standards.
For the commercial production of electronic devices, EMI measurements are inevitable but time consuming. Automation helps to optimize a measurement process [1] and offers some reduction in the production time. However, it is still limited by the superheterodyne principle of traditional receivers. The introduction of new faster EMI measuring principles would bring significant benefits in time to market and costs and could probably support the idea of just in time production [2].

The time domain EMI (TDEMI) system [3] was introduced quite recently. It is based on a multiresolution analog-to-digital converter (MRADC) technology, which engages several parallel input channels to achieve the

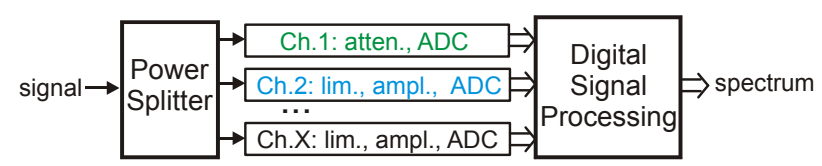

Figure 1. Block structure of a TDEMI system. 

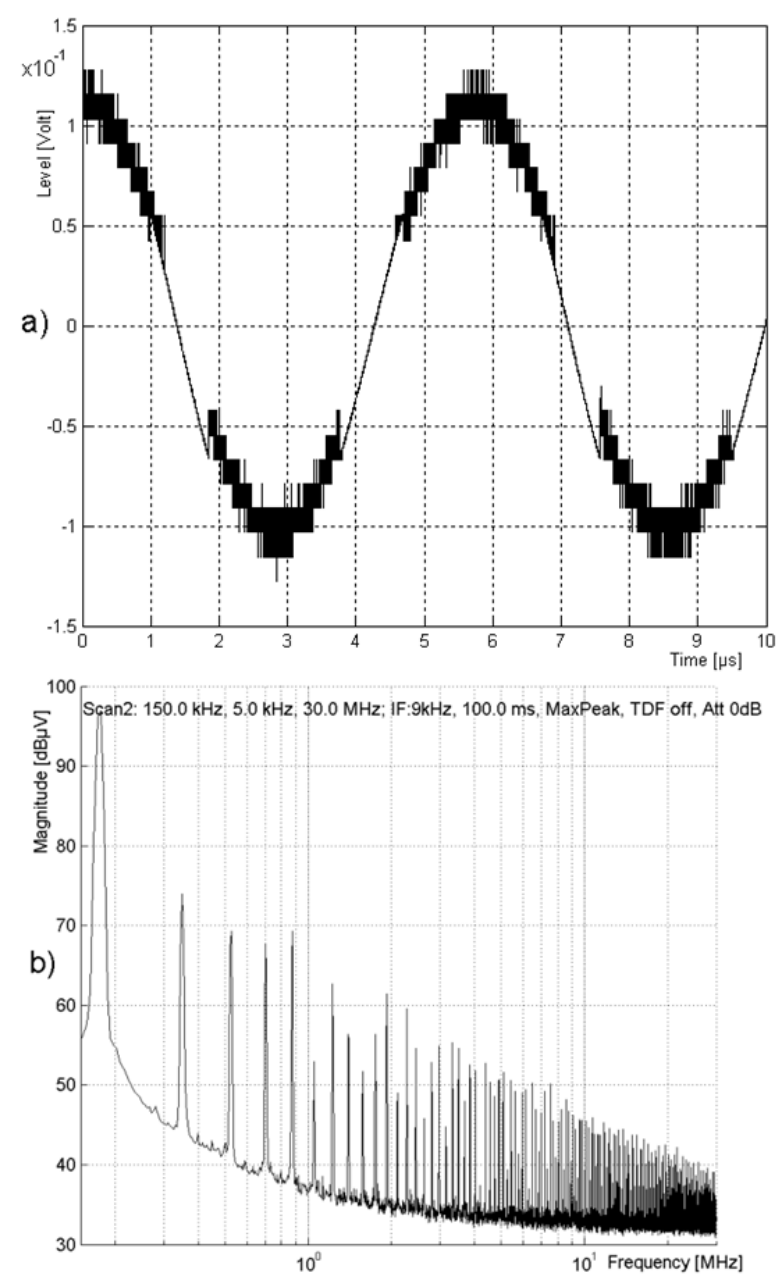

Figure 2. Experimental harmonic signal measured by a real TDEMI system: a) time representation inside the TDEMI unit; b) measured spectrum.

required quality of the system. The block structure of a TDEMI device is depicted in Figure 1. The power splitter distributes the analog signal to all paths and 3 channels are common at present. All ADCs are very fast sampling ones of the same type and range. Separate amplifiers/attenuators provide different signal ranges and voltage resolutions of individual channels. Channel 1 covers the entire range. The range of the next channel is part of the previous one and therefore the input signal will likely exceed the range of subsequent channels. Here, the limiter protects the ADC input from overvoltage. All channels are simultaneously sampled and converted by identical 8 or 10 bit very fast flash ADCs. The final discrete value is created by extracting the output from that $\mathrm{ADC}$ offering the best resolution but with the range still covering the actual input value. Short time Fast Fourier Transform (FFT) is finally applied to the sampled data [4] to obtain one representation of the whole frequency spectrum.

TDEMI devices process the complete spectral content at a time and the parallel structure of the MRADC should still allow a high dynamic range of measurements [5]. Some influence of the quantization process on the measured spectra [6] remains also for the MRADC [7]. However practical experience shows that there are more serious error sources in real systems [8]. It is hardly possible to avoid the offset or phase shift between parallel input channels likewise the gain error differences. Similar problems occur in ADC systems with time interleaving or with reconfiguration structure [9] where offset or gain mismatch of parallel channels degrades the overall performance. So serious signal discontinuities could arise in points where the system switches between channels. Spurious spectrum components generated by those discontinuities significantly restrict the spurious free dynamic range (SFDR) of a real TDEMI device.

The paper deals with identification of TDEMI device errors and with the design of a precise error model. Imperfections of a real TDEMI system will be explained in chapter 2. A pulse model of the dominant error of spectrum measurements will be discussed in chapter 3. In the next chapter the identification process of channel discrepancy will be presented which offers finding model parameters. Finally we will be able to compare the proposed error model with experimental results.

\section{IMPERFECTIONS OF REAL TDEMI SYSTEM}

The concept of an MRADC lies in the use of several parallel ADCs each with uniform but different quantization steps. The minimum quantization step for the multiresolution quantization corresponds to the step of the channel with the lowest range. Actually, the system range is divided into subranges with different quantization steps. Even if perfectly realized such system generates disturbance in the quantized spectrum, as was shown in [7] for a harmonic signal. In the experimental waveform obtained from the TDEMI device output and plotted in Figure 2a we can observe that in distant parts from the zero axis there is a significant quantization error. However, in a real system there are more serious error sources. The main task of the analog input circuits is to split the input signal into multiple paths with precisely set gains. This is a non-trivial task because ideally a uniform amplification level should be achieved for the frequency range up to several $\mathrm{GHz}$ without introducing a different phase shift between channels. Channel mismatch compensation techniques have been developed especially for time interleaved ADCs [10]. On the other hand, publications describing properties of TDEMI measurement systems are dealing mostly with a perfectly matched power splitter and a digital processing module. In a real system amplitude and phase frequency characteristics in each channel are not perfectly matched over the whole frequency range. Moreover it is hardly possible to avoid the offset and slope difference between channels as the gain must be different in each channel. In the example of the time representation of the signal (Figure 2a, frequency $175 \mathrm{kHz}$ and amplitude $0.128 \mathrm{~V}$ ) such discontinuities are especially evident in the negative portion of the waveform around the threshold of ca. $-60 \mathrm{mV}$ between the upper two channels of a three-channel MRADC. Error components resulting from those discontinuities significantly restrict the SFDR of the spectrum measurement. We will demonstrate that channel discrepancy is the main contribution to spurious 


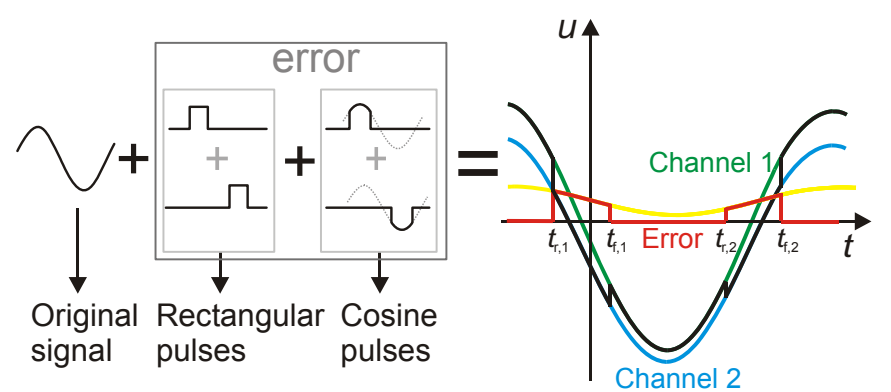

Figure 3. Basic idea of the pulse error model.

components visible in the measured spectrum depicted in Figure $2 \mathrm{~b}$ obtained for the same harmonic input signal.

Experiments suggest that the main spurious components of TDEMI measurement results are caused by discontinuities in the time domain signal representation. An harmonic input signal may be considered as suitable for modeling the measured interference of devices operating on the switched mode power supply principle, where the disturbance is like a mixture of sinusoids. We will assume a two-channel MRADC as the major influence seems to be the mismatch between the upper two channels. Differences between channels result in disturbances similar to timedomain error pulses (and not only for the harmonic input) like sketched in Figure 3. Discontinuities present in the waveform reconstructed from the sequence of samples obtained by the MRADC could be modeled as an additive impulse error signal. Then, the spectrum of the impulse disturbance could be used to precisely model the resulting spurious spectral components. This is the fundamental assumption used in our analysis. Two rectangular pulses represent the offset between channels while two cosine shaped pulses describe the gain and phase discrepancy.

\section{ANALYTICAL EXPRESSION OF ERROR MODELS}

The proposed idea of pulse representation of errors can be utilized in the simulation model or for the evaluation of the analytical model. Two error pulses of the model per period of the input signal enable precise estimation of the real error if proper shapes of pulse-forming waveforms are used. For an harmonic input signal the harmonic waveform (yellow "cosine original" in Figure 3) with the right offset, gain and phase composes pulses for such accurate simulation model. Compared to a real system the model neglects quantization errors, however, it is precise for the estimation of the distortion caused by the discrepancy between channels and can be used as a reference for other approximative models.

In a TDEMI system the measured spectrum is digitally evaluated from samples. Digital systems calculate the spectrum by Discrete Fourier Transform (DFT) usually using an FFT algorithm. DFT output approximates coefficients of Fourier series, which decomposes the given periodic function $u(t)$ with frequency $f_{0}$ into the sum of harmonic functions. Therefore, speaking about spectrum, we are trying to find coefficients $U_{\mathrm{n}}=a_{\mathrm{n}-\mathrm{j}} b_{\mathrm{n}} \quad(n=0,1,2$; $b_{0}=0$ ) of the Fourier series.
Table 1. Auxiliary functions.

\begin{tabular}{ll}
\hline Name & Formula \\
\hline $\mathrm{CpS}_{\mathrm{n}}(\omega, t, \varphi)$ & $\cos (\omega t+\varphi) \sin (n \omega t)$ \\
$\mathrm{SpC}_{\mathrm{n}}(\omega, t, \varphi)$ & $\sin (\omega t+\varphi) \cos (n \omega t)$ \\
$\mathrm{CpC}_{\mathrm{n}}(\omega, t, \varphi)$ & $\cos (\omega t+\varphi) \cos (n \omega t)$ \\
$\mathrm{SpS}_{\mathrm{n}}(\omega, t, \varphi)$ & $\sin (\omega t+\varphi) \sin (n \omega t)$ \\
\hline
\end{tabular}

The error model is composed of two rectangular and two cosine pulses and at first we need to find the spectral components of all the pulses. Rectangular pulses describe the offset between channels, i.e. the offset of the erroneous cosine original (the offset of the yellow waveform in Figure 3). The zero coefficient of one rectangular pulse is

$a_{\mathrm{RP}, 0}\left(\omega_{0}, A, t_{\mathrm{r}}, t_{\mathrm{f}}\right)=\frac{A}{2 \pi} \omega_{0}\left(t_{\mathrm{f}}-t_{\mathrm{r}}\right)$

while for $n>0$ we can write

$a_{\mathrm{RP}, n}\left(\omega_{0}, A, t_{\mathrm{r}}, t_{\mathrm{f}}\right)=\frac{A}{2 \pi n}\left[\sin \left(n \omega_{0} t_{\mathrm{f}}\right)-\sin \left(n \omega_{0} t_{\mathrm{r}}\right)\right]$,

$b_{\mathrm{RP}, n}\left(\omega_{0}, A, t_{\mathrm{r}}, t_{\mathrm{f}}\right)=-\frac{A}{2 \pi n}\left[\cos \left(n \omega_{0} t_{\mathrm{f}}\right)-\cos \left(n \omega_{0} t_{\mathrm{r}}\right)\right]$,

where $\omega_{0}$ is the angular frequency of pulses, $A$ is the amplitude of the rectangular pulse, $t_{\mathrm{r}}$ and $t_{\mathrm{f}}$ is the time of rising and falling edge. Cosine pulses of the model involve the gain and phase discrepancy. Spectral components of one cosine pulse are

$$
\begin{aligned}
& a_{\mathrm{CP}, 0}\left(\omega_{0}, A, t_{\mathrm{r}}, t_{\mathrm{f}}, \varphi\right)=\frac{A}{2 \pi}\left[\sin \left(\omega_{0} t_{\mathrm{f}}+\varphi\right)-\sin \left(\omega_{0} t_{\mathrm{r}}+\varphi\right)\right] \\
& a_{\mathrm{CP}, 1}\left(\omega_{0}, A, t_{\mathrm{r}}, t_{\mathrm{f}}, \varphi\right)=\frac{A \omega_{0}\left(t_{\mathrm{f}}-t_{\mathrm{r}}\right)}{4 \pi} \cos (\varphi) \\
& +\frac{A}{8 \pi}\left[\sin \left(2 \omega_{0} t_{\mathrm{f}}+\varphi\right)-\sin \left(2 \omega_{0} t_{\mathrm{r}}+\varphi\right)\right] \\
& b_{\mathrm{CP}, 1}\left(\omega_{0}, A, t_{\mathrm{r}}, t_{\mathrm{f}}, \varphi\right)=-\frac{A \omega_{0}\left(t_{\mathrm{f}}-t_{\mathrm{r}}\right)}{4 \pi} \sin (\varphi) \\
& -\frac{A}{8 \pi}\left[\cos \left(2 \omega_{0} t_{\mathrm{f}}+\varphi\right)-\cos \left(2 \omega_{0} t_{\mathrm{r}}+\varphi\right)\right] \\
& \text { and for } n>1 \\
& a_{\mathrm{CP}, n}\left(\omega_{0}, A, t_{\mathrm{r}}, t_{\mathrm{f}}, \varphi\right)= \\
& \frac{A}{2 \pi\left(n^{2}-1\right)}\left[n \mathrm{CpS}_{\mathrm{n}}\left(\omega_{0}, t_{\mathrm{f}}, \varphi\right)-\operatorname{SpC}_{\mathrm{n}}\left(\omega_{0}, t_{\mathrm{f}}, \varphi\right)\right] \\
& -\frac{A}{2 \pi\left(n^{2}-1\right)}\left[n \mathrm{CpS}_{\mathrm{n}}\left(\omega_{0}, t_{\mathrm{r}}, \varphi\right)-\operatorname{SpC}_{\mathrm{n}}\left(\omega_{0}, t_{\mathrm{r}}, \varphi\right)\right] \\
& b_{\mathrm{CP}, n}\left(\omega_{0}, A, t_{\mathrm{r}}, t_{\mathrm{f}}, \varphi\right)= \\
& -\frac{A}{2 \pi\left(n^{2}-1\right)}\left[n \operatorname{CpC}_{\mathrm{n}}\left(\omega_{0}, t_{\mathrm{f}}, \varphi\right)+\operatorname{SpS}_{\mathrm{n}}\left(\omega_{0}, t_{\mathrm{f}}, \varphi\right)\right] \\
& +\frac{A}{2 \pi\left(n^{2}-1\right)}\left[n \operatorname{CpC}_{\mathrm{n}}\left(\omega_{0}, t_{\mathrm{r}}, \varphi\right)+\operatorname{SpS}_{\mathrm{n}}\left(\omega_{0}, t_{\mathrm{r}}, \varphi\right)\right]
\end{aligned}
$$

In this case, besides the frequency, times of edges and the amplitude of the cosine original $A$ also its phase $\varphi$ enters the argument of expressions. For $n>1$ the formulas are more complicated. Therefore we have defined auxiliary 
functions (see Table 1) which have allowed those simplified notations (7)(8).

To write the final expression of spectral error components we follow the idea of two actual error pulses mathematically represented by two pairs of pulses. The time of the rising and falling edge is $t_{\mathrm{r}, 1}, t_{\mathrm{t}, 1}$ for the first error pulse and $t_{r, 2}, t_{t, 2}$ for the second. Both error pulses are described by the sum of the rectangular pulse and the cosine pulse to cover offset, gain and phase error. Considering that $a_{\mathrm{n}}$ is the real part and $-\mathrm{j} b_{\mathrm{n}}$ the imaginary part of the spectral coefficient $U_{n}$ (use index RP and $\mathrm{CP}$ for rectangular and cosine pulse) the error model states

$$
\begin{aligned}
& \mathbf{U}_{\mathrm{E}, n}\left(\omega_{0}, A_{\mathrm{O}}, A_{\mathrm{CO}}, t_{\mathrm{r} 1}, t_{\mathrm{f} 1}, \varphi_{\mathrm{CO}}\right)= \\
& \mathbf{U}_{\mathrm{RP}, n}\left(\omega_{0}, A_{\mathrm{O}}, t_{\mathrm{r} 1}, t_{\mathrm{f} 1}\right) \\
& +\mathbf{U}_{\mathrm{RP}, n}\left(\omega_{0}, A_{\mathrm{O}}, t_{\mathrm{r} 2}, t_{\mathrm{f} 2}\right) \\
& +\mathbf{U}_{\mathrm{CP}, n}\left(\omega_{0}, A_{\mathrm{CO}}, t_{\mathrm{r} 1}, t_{\mathrm{f} 1}, \varphi_{\mathrm{CO}}\right) \\
& +\mathbf{U}_{\mathrm{CP}, n}\left(\omega_{0}, A_{\mathrm{CO}}, t_{\mathrm{r} 2}, t_{\mathrm{f} 2}, \varphi_{\mathrm{CO}}\right)
\end{aligned}
$$

The amplitude $A$ o of rectangular pulses is just the offset of channel 2 towards channel 1 . The amplitude and phase of cosine pulses are taken from the erroneous cosine original and could be calculated using phasor rules. If the reference channel 1 has ideal parameters (zero phase and gain of one) the ideal output waveform is $A_{1} \cos (\omega 0 \mathrm{t})$ or $A_{1} \angle 0$ in the phasor notation. The amplitude $A_{1}$ is the amplitude of the input signal or rather the amplitude of the signal obtained solely from channel 1 . Then the phasor of the erroneous cosine original is

$$
A_{\mathrm{CO}} \angle \varphi_{\mathrm{CO}}=A_{1} \angle 0-A_{1} G_{2} \angle \varphi_{2}
$$

where $G_{2}$ is the gain of channel 2 and $\varphi_{2}$ is its phase shift.

We can make some further simplifications in the proposed model (9). As we assume ideal parameters of channel 1 and if the switching between channels would ideally work according to channel 1 , the rectangular pulses in equation (9) will be the same. Then they could be replaced by one rectangular pulse signal with double frequency for even $n$ and totally omitted for odd $n$ [8]. In other words, we could expect that the offset discrepancy will impact only even error harmonics. On the other hand if the offset is the dominant mismatch parameter a simplified model can be used with only rectangular pulses (REC model in [8]). Then, the amplitude $A$ o should be calculated as mean of the samples within the real error pulse and separately for each rectangular pulse if the real error pulses are not perfectly square.

\section{ERROR IDENTIFICATION}

To be able to apply the proposed model to experimental data we need to extract main error parameters from the measured waveform (Figure 2a). The parameters requested by the model are the precise signal frequency $f_{0}$, the real signal amplitude $A_{1}$, the offset between channels $A_{\circ}$, the gain error described by gain $G_{2}$ of the second channel and the phase shift between channels $\varphi_{2}$. Also times of rising and falling edges of both error pulses $t_{r, 1}, t, 1, t_{r, 2}, t, 2$ should be estimated from the experimental data. To obtain precise results and a repeatable formal process of the estimation we proposed a special procedure of the error identification. It can be summarized into three main steps: 1 . Identification of edges; 2 . Curve fitting; 3 . Determination of parameters of the error model.

\subsection{Identification of edges}

Moments of error pulse edges are points where the system switches from one channel to another. From Figure 2 it is obvious that noise present in the sampled waveform is a good indication of the channel. Unfortunately, we are able to obtain only a screenshot from the commercial TDEMI unit. Therefore we needed at first to identify data from the original bitmap. Our method based on colour shades provided data drawn black in Figure 4a. The noise was slightly suppressed here by the data extraction algorithm. However, the difference between channels is still apparent. So, the identification of edges is based on signal noise and is done in the following steps.

\section{Noise identification}

To extract noise from the measured signal we smoothed the waveform using moving average (dark gray line in Figure 4a). Subsequently we separated the noise by subtracting the smoothed waveform from its original. The noise itself is depicted light gray in Figure $4 a$ or in Figure $4 \mathrm{~b}$ at a better scale.

\section{Noise thresholding}

Parts of the noise data of low and high variance relate to the currently used input channel. To identify moments of channel change we need to find points of the variance change. The amount of noise in the actual time point was estimated in Figure $4 \mathrm{a}$ from the moving root mean squared value (MRMSN, turquoise solid curve). Finally by thresholding (with $0.5 \mathrm{mV}$ ) we identified edges depicted by turquoise dashed verticals.

\section{Edge times adjustment}

Edges found by MRMSN thresholding are visibly shifted away from the exact points of noise variance changes. This follows from the nature of squaring which favors higher values. Therefore we continued in searching of precise edge times in the vicinity of previously found approximate edges. This time we will use the moving average from the absolute noise values (MAAN, blue solid curve in Figure 4a and Figure 4b). MAAN does not offer as good distances for thresholding as MRMSN. However, in points of exact edges local extremes are located. Indeed there is a good chance that exactly in the point of channel change a significant noise value occurs due to the jump in the final waveform caused by the channel discrepancy. To increase the impact of the actual noise value we used a weighted averaging for the MAAN evaluation. Sometimes a special process of final edge discrimination could be needed if some fake edges were also found. In both Figure $4 \mathrm{a}$ and Figure $4 \mathrm{~b}$ the precise adjusted edges are plotted as blue dashed verticals.

\subsection{Curve fitting}

After identification of edges we are able to separate parts of the waveform samples corresponding to the first or second channel. (At this point we also set the time of the 

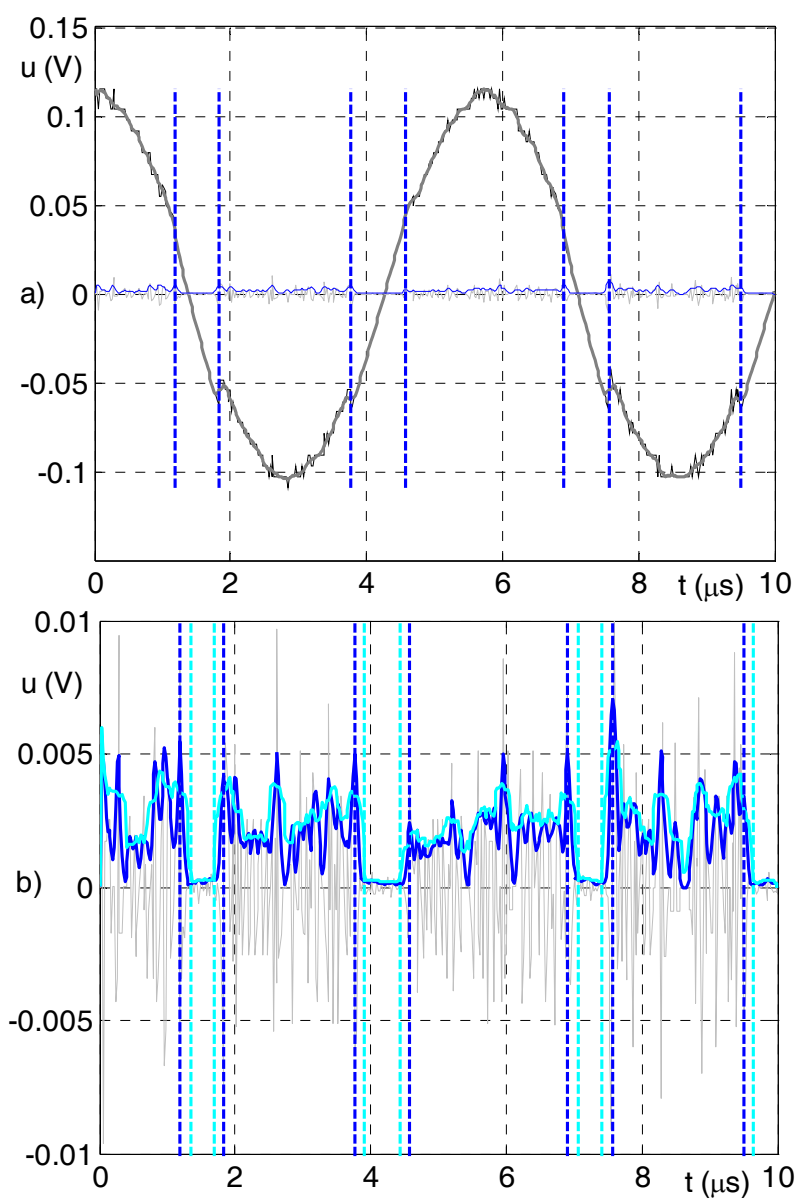

Figure 4. Detection of times of edges. a) original and smoothed MRADC output signal with detected switching points; b) noise data used for thresholding and adjusting of edges.

first sample to zero.) The basic function for fitting is cosine and we want to identify four parameters. The frequency should be the same for both channels while the amplitude, offset and phase are independent.

\section{Channel data separation}

Points of separation are the previously adjusted edge moments. In some cases there are fluctuations near the switching point. Therefore it is possible and often suitable to omit a few samples from each side of the given edge. We also need to set the channel order: channel 1 data are green and channel 2 data are blue.

\section{Setting initial conditions}

Nonlinear curve fitting methods require initial data for starting the numeric algorithm. The main input parameter here is a starting frequency which might be the set frequency of the test signal. Knowing the frequency the other starting parameters (amplitude, offset, phase) could be calculated by linear fitting applied on the whole data set.

\section{Nonlinear fitting}

To identify the frequency of the cosine original we need to use a nonlinear curve fitting algorithm. Data from channel 2 contain less noise, therefore we considered the channel 2 output as more suitable for determination of the frequency. We recommend a least squares method for the fitting and we used unweighted non-robust least squares implemented within Matlab statistic library functions

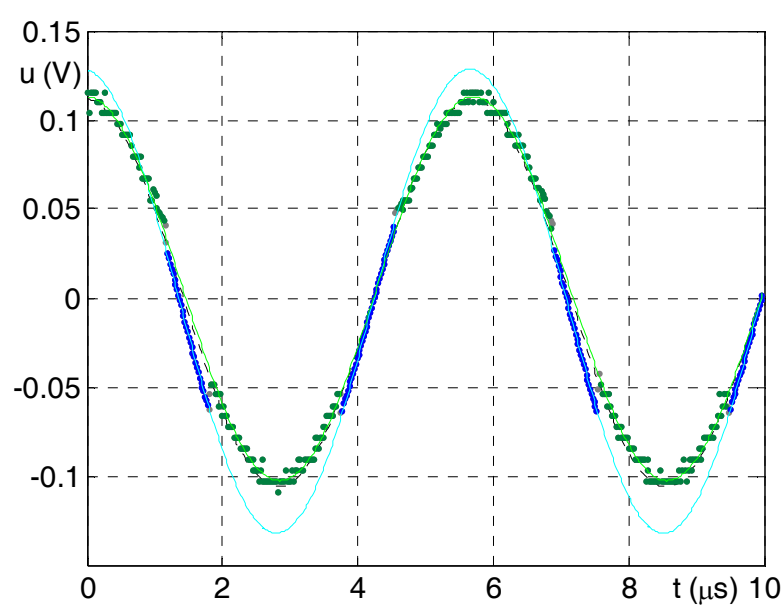

Figure 5. Curve fitting performed separately for the data from the first and second channel.

(Gauss-Newton algorithm, [11]). From the procedure also the amplitude, offset and phase of the signal from channel 2 are obtained. The cosine fitted to the channel 2 data is depicted as turquoise solid waveform in Figure 5.

\section{Linear fitting}

If we already know the frequency of the cosine signal being identified a simple linear curve fitting algorithm can be used. The amplitude, offset and phase of the cosine signal approximating channel 1 data could be found from the solution of a pertinent linear system of equations using matrices and Matlab. In Figure 5 this fitted function is depicted as a light green solid waveform.

\subsection{Determination of parameters of the error model}

Identified parameters should be further accommodated for the error model. Note that we want to use the channel 1 signal as the reference and therefore e.g. the time values have to be adjusted in the way that we force the zero offset and phase of this channel. Discrepancy parameters of channel 2 will be related to channel 1.

\section{Parameters of input reference signal}

The frequency identified during the curve fitting process is simply the frequency fo of the input signal which enters the error model. In our case we calculated $f_{0}=174.832 \mathrm{kHz}$. As channel 1 is the reference one, amplitude $A_{1}=0.1079 \mathrm{~V}$ identified from this channel will be assigned also to the input or ideal output signal.

\section{Parameters of channel discrepancy}

By comparison of both channels we can calculate for channel 2: the offset $A \circ=-6.9920 \mathrm{mV}$, its gain $G_{2}=1.2078$ and the phase $\varphi_{2}=3.0647^{\circ}$.

\section{Uniform set of edge times}

From previously identified edge moments we need to solve relative times toward the start of a given period of the reference signal. The same edge could be identified several times if more input periods are contained in a test interval. Four edge times per period are expected for the cosine input signal and from Figure $4 \mathrm{~b}$ one can notice that the first 


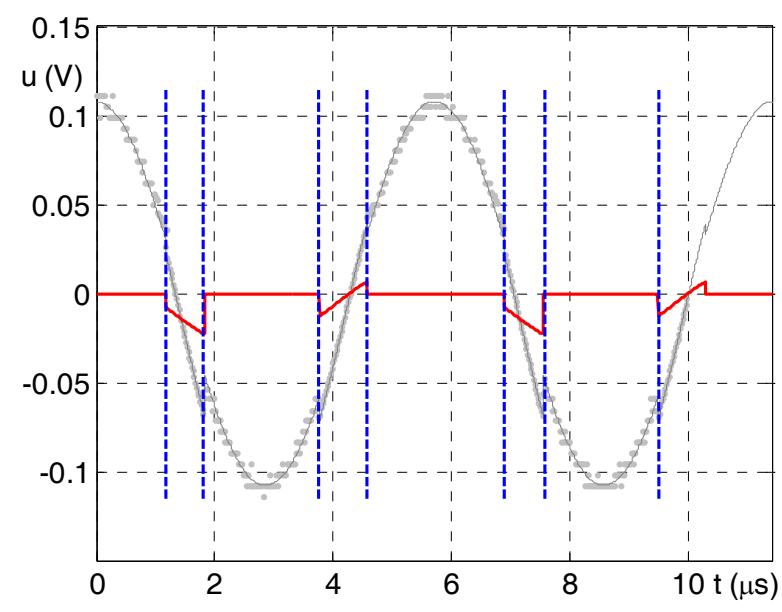

Figure 6. Time representation of simulated error pulse signal and distorted MRADC output waveform.

three are identified two times. In that case we can calculate the final time of a given edge as a simple mean of all corresponding relative edge times. Our final uniform set of four edges is: $t_{\mathrm{r}, 1}=1.1795 \mu \mathrm{s}, \quad t_{\mathrm{f}, 1}=1.8320 \mu \mathrm{s}$, $t_{\mathrm{r}, 2}=3.7667 \mu \mathrm{s}, t_{\mathrm{f}, 2}=4.5713 \mu \mathrm{s}$.

\section{RESULTS AND DISCUSSION}

Parameters of the input signal, the channel discrepancy and switching times were used in the simulation as well as in the analytical model. In the time domain we used the simulation model. Error pulses obtained from the model for two periods of the input signal are depicted in red in Figure 6. The MRADC output signal disturbed by those pulses is in dark gray. The light gray dots in the background are original experimental data only slightly shifted to impose ideality of channel 1 . The model waveform visibly fits the experimental data in the time range where they are available. Blue dashed verticals represent all 7 edges identified from experiments and adjusted for the used point of zero time. In the simulation model we used one set of four edges for both periods. Therefore e.g. the time of the second edge of red error pulses $(t, 1=1.8320 \mu \mathrm{s})$ is the mean from time shifts of the second and sixth blue vertical from the start of the given period.

After the identification of parameters entering the pulse model and after the verification in the time domain we are ready to confront model results with the experimental spectrum. Error spectral components evaluated from the analytical pulse model (9) are depicted in Figure 7a as red circles. Only the gray " $x$ " represents the measured component i.e. the amplitude of the input harmonic signal. The simulation error spectrum (gray lines with dot markers) is almost identical with results of analytical expressions. Black circles are original experimental error spectral components (from Figure 2b). Apparently there is a visible similarity between the model and experimental results. We can observe a little shift of experimental points from the theoretical ones, however the overall trend or the
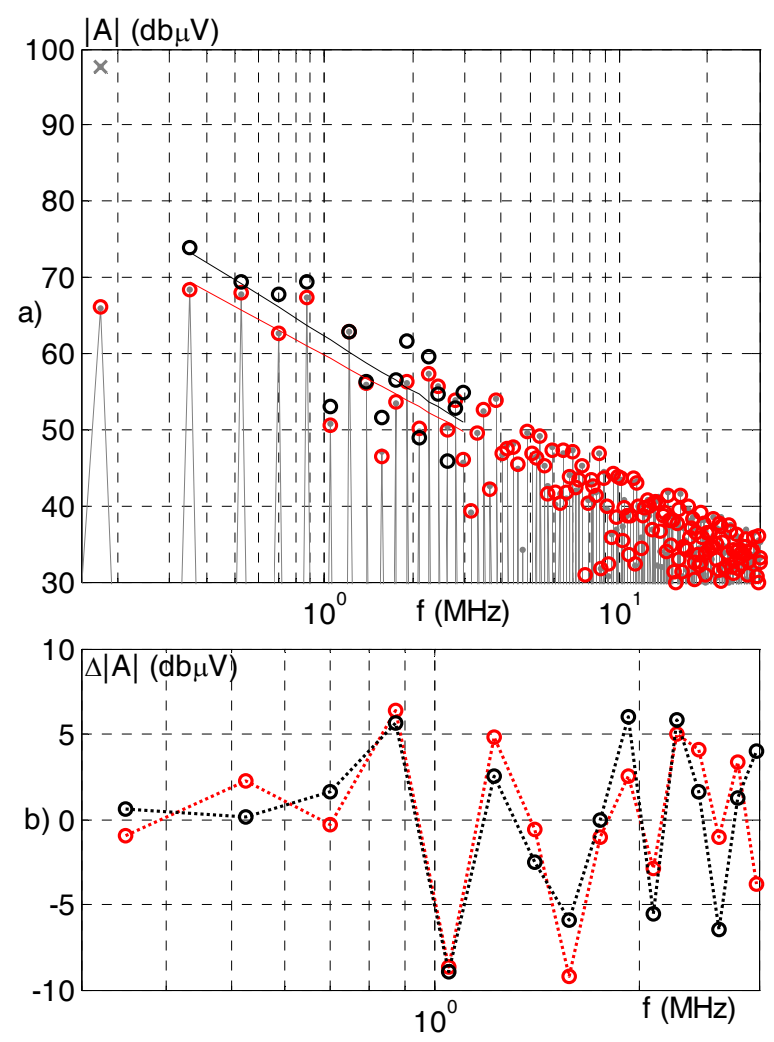

Figure 7. Comparison of experimental error spectrum and error components obtained from the pulse model: a) experimental, simulation and theoretical spectra; b) differences from the line fit.

shape of fluctuations is satisfactory estimated by theoretical expressions. The correlation coefficient is as high as 0.9130 in the compared range of approximately one frequency decade. Note that if we used only rectangular pulses in the analytical model the correlation was 0.6928 . If we compare experimental results to the own line fit (black solid line), the correlation coefficient is $\mathbf{0 . 8 1 8 8}$. So the overall decay of the error spectrum is not the whole similarity and the number 0.9138 embraces also the similarity in fluctuations.

To compare only the shape of fluctuations we decided to subtract the overall decay from spectral components. In Figure $7 \mathrm{a}$ the lines fitted trough the points are drawn as black or red solid lines for experimental or model results. Subsequently in Figure $7 \mathrm{~b}$ only differences from this overall decay are depicted. Experimental data are in black here while the model results are red again. The similarity is more visible now and the calculated correlation coefficient of 0.7614 is not influenced by the decay of spectral components with rising frequency.

\section{CONCLUSIONS}

The precise pulse error model for the estimation of error spectral components disturbing measurements performed by a time domain electromagnetic interference (TDEMI) measuring system was presented in the paper. The analytical model was expressed for a two channel system and the harmonic input signal. Furthermore a consistent process of the error parameters identification from experimental data has been proposed based on the signal 
noise. The comparison of model and experimental results has shown that the channel discrepancy represented by the analytical model has a dominant impact on error components occurring in the measured spectrum. Shape changes in the experimental spurious components were adequately reproduced by the model while the overall decay was similar too. Differences and little shift between compared values could be explained by simplifications used in the model, a limited interval of experimental timedomain data processed during the identification phase, windowing and asynchronous sampling in a real TDEMI unit, etc.

The designed model could be useful for the further development and improvements of the technology behind TDEMI. It could help to find right switching points for expected type of the input signal or indicate critical conditions for the measurement method of TDEMI. The proposed process of the error parameter identification helps to find realistic values and intervals of parameters describing the discrepancy. The estimation of the error behaviour is a necessary step towards future searching for methods of error correction.

\section{ACKNOWLEDGEMENT}

Work presented in this paper was supported by the Slovak Research and Development Agency under grant No. APVV-0333-11 and by the Slovak Grant Agency VEGA under grant No. 1/0963/12.

\section{REFERENCES}

[1] O. Čičáková, P. Štefanička, Efficiency of automated measurement systems, Measurement Science Review, 6 (4) (2006) pp. 50-53.

[2] B. Moric Milovanovic, B. Sisek, M. Kolakovic, Just in time concept as a mean for achieving competitive advantage in the virtual economy, Proceedings of 22th International DAAAM Symposium, DAAAM International Vienna, Austria, 2011, pp. 1105-1106.
[3] S. Braun, P.Russer, The dynamic range of a time-domain EMI measurement system using several parallel analog to digital converters, Proceedings of the 16th International Zurich Symposium on Electromagnetic Compatibility, Swiss Federal Institute of Technology Zurich, Switzerland, 2005, pp. 203-208.

[4] F. Krug, D. Mueller, P. Russer, Signal processing strategies with the TDEMI measurement system, IEEE Transactions on Instrumentation and Measurement, 53 (5) 2004, pp. 14021408.

[5] S. Braun, P. Russer, A low-noise multiresolution highdynamic ultra-broad-band time-domain EMI measurement system, IEEE Trans. on Microwave Theory and Techniques, 53 (11), 2005, pp. 3354- 3363.

[6] T.A.C.M. Claasen, A. Jongepier, Model for the power spectral density of quantization noise, IEEE Transactions on Acoustics, Speech and Signal Processing, ASSP-29 (4), 1981, pp. 914-917.

[7] M. Kamenský, K. Kováč, G. Války, Improvement in spectral properties of quantization noise of harmonic signal using multiresolution quantization, IEEE Transactions on Instrumentation and Measurement, 61 (11), 2012, pp. 28882895.

[8] M. Kamenský, K. Kováč, G. Války, Model of errors caused by discrepancies of Input channels in multiresolution ADC. IMEKO 2013 : TC-4 Symposium on Measurements of Electrical Quantities and 17th IEADC Workshop on Advances in Instrumentation and Sensors Interoperability, Barcelona, Spain, 2013, pp. 178-183.

[9] W. Carvajal, W. Van Noije, An optimization-based reconfigurable design for a 6-bit $11-\mathrm{MHz}$ parallel pipeline ADC with double-sampling S\&H, International Journal of Reconfigurable Computing, Article ID 786205, 2012, 17 pages.

[10] H.A. Nawaz, A. Sharif, M. Sharif, Comparative survey on time interleaved analog to digital converter mismatches compensation techniques, Research Journal of Recent Sciences, 2 (9), 2013, pp. 95-100.

[11] H. Wen, J. Ma, M. Zhang, G. Ma, The Comparison Research of Nonlinear Curve Fitting in Matlab and LabVIEW. 2012 IEEE Symposium on Electrical \& Electronics Engineering (EEESYM), Kuala Lumpur, Malaysia, 2012, pp. 74-77. 